\title{
Quality Control of High-Speed Photon Detectors
}

\author{
Yusuke Inome ${ }^{1,2^{*}}$, Yuji Sunada ${ }^{3}$, Yuki Choshi $^{2}$ Masao Ichida ${ }^{1}$, Razmik Mirzoyan $^{4}$, Hideyuki Ohoka ${ }^{2}$, Takayuki Saito $^{2}$, Kinji $^{2}$ \\ Tamura $^{1}$, Masahiro Teshima ${ }^{2,4}$, and Tokonatsu Yamamoto ${ }^{1}$ \\ ${ }^{1}$ the Faculty of Science and Engineering, Konan University, Kobe 658-8501, Japan \\ ${ }^{2}$ the Institute of Cosmic Ray Research, University of Tokyo, Kashiwa, Chiba 277-8582, Japan \\ ${ }^{3}$ the Graduate Schoole of Science and Engineering, Saitama University, Saitama 338-8570, Japan \\ 4the Max Planck Institute for Physics, D-80805 Munich Germany
}

\begin{abstract}
High-speed-photon detectors are some of the most important tools for observations of high energy cosmic rays. As technologies of photon detectors and their read-out electronics improved rapidly, the time resolution of some cosmic ray detectors became better than one nanosecond. To utilize such devices effectively, calibrations using a short-pulse light source are necessary. We have developed a pulsed laser of 80 picosecond width and adjustable peak intensity up to $100 \mathrm{~mW}$. This pulsed laser is composed of a simple electric circuit and a laser diode. Details of this pulsed laser and its application for quality controls of photon detectors are reported in this contribution.
\end{abstract}

\section{Introduction}

Short pulses of light, such as Cherenkov or scintillation light, are one of the important signals in high-energy experiments. A high-energy particle travels at essentially the speed of light. It can be accompanied by the Cherenkov photons which are emitted from the charged particle itself in a material. The Cherenkov photons become coherent with the photons from later emissions. As a result, the bunch of photons arrives at the detector simultaneously. Therefore, the detectors to observe such phenomena are required to have high time resolutions [1]. Photomultiplier tubes (PMTs) are widely used for the measurements of faint light and some of these can have a time resolution of a few nanoseconds. Biplanar phototubes can measure photons with a risetime of less than a hundred picoseconds. Recently a semiconductor device called Multi-Pixel Photon Counter (MPPC) or Silicon Photomultiplier (SiPM) has become popular and has even faster response time. Short-pulse light sources are necessary to calibrate such devices. In general, a light source with pulse width less than one nanosecond can be quite expensive. However, electronics components with high frequency and high driving power are going on the market at even lower prices. Utilizing such components, electronics pulses of few nanosecond width are available using a simple and low-cost circuit.

A Laser Diode (LD) is suitable for such a light source. The intensity of light emission from a LD can easily be modulated by modulating the electric current applied to the LD. Because of its high-speed response to the applied current, the LD is widely used for telecommunications and measurements. A short light pulse can simply be produced by applying a short electrical pulse to the LD [2]. Optimizing the width and amplitude of the electrical pulse, the width of the laser pulse can be significantly shorter than that of applied.

In this study we describe a design and optimization of a pulsed laser which stably emits laser pulses with 80 picoseconds in FWHM using simple and low-cost electronics components. We explain the principle of the pulsed laser system in section 2, the optimized electronics circuit in section 3, an application of the pulsed laser to a calibration of a photon detector in section 4 , and finally we summarize these results.

\section{Principle of Pulsed Laser}

A LD is a semiconductor laser. Charged carriers (electrons and holes) are pumped into the $\mathrm{P}$ and $\mathrm{N}$ region of its p-intrinsic-n (PIN) structure when a current is applied. Photons are generated in the intrinsic region by "spontaneous emissions" which are caused by recombination, or annihilation, of the carriers. The carrier density $n$ is increased by the applied current. Then the photon density $S$ is gradually increased by spontaneous emission. The photons induce "stimulated emissions" which generate additional photons and consume the carriers. The relationship of $S$ and $n$ in a LD can be described by the following rate equation

$$
\begin{aligned}
& \frac{d n}{d t}=\frac{J}{e d}-\frac{n}{\tau_{c}}-g \cdot n \cdot S \\
& \frac{d S}{d t}=C \frac{n}{\tau_{c}}-\frac{S}{\tau_{p}}+g \cdot n \cdot S
\end{aligned}
$$

where $J$ is the applied current density, $e$ is the elementary charge, $d$ is the thickness of the active

\footnotetext{
* Corresponding author: sinome05@gmail.com
} 
region, $\tau_{0}$ is the carrier lifetime, $\tau_{\mathrm{p}}$ is the photon lifetime, $C$ is

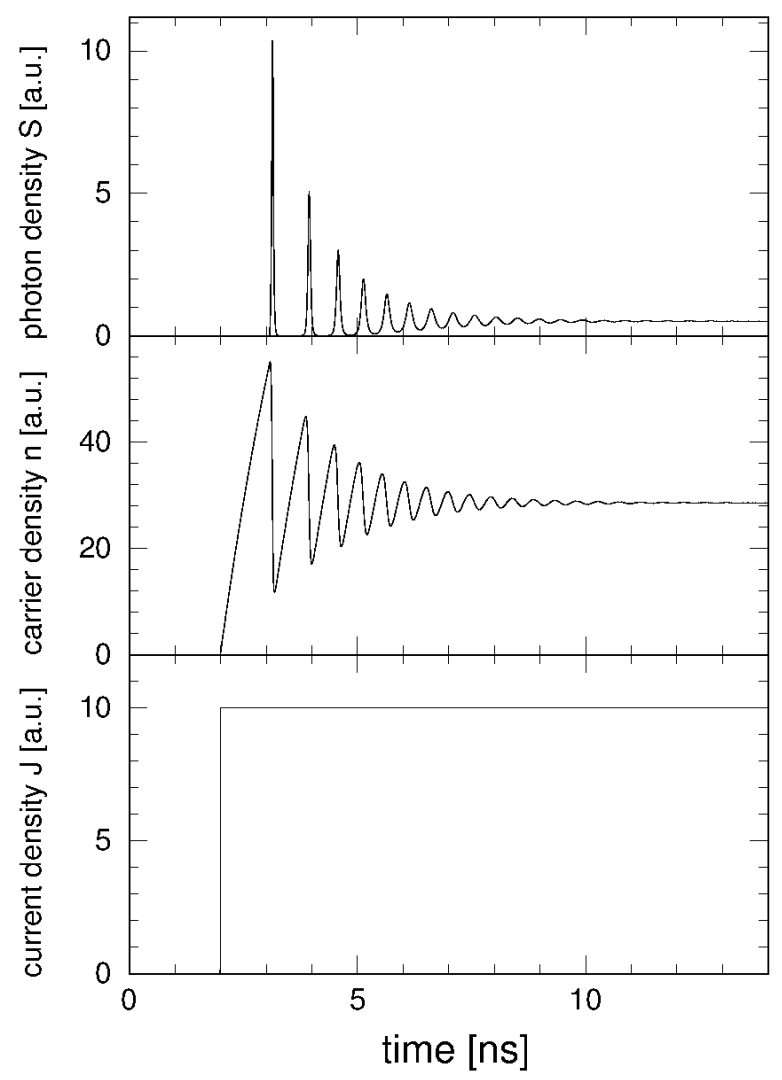

Fig. 1. Simulation of laser emission from a LD. An electric current with step function is applied. Applied current density $\mathrm{J}$ (bottom), carrier density $n$ (middle), and photon density $S$ (upper) are shown as a function of time. $\tau_{\mathrm{p}}$ and $\tau_{\mathrm{c}}$ are assumed to be 10 picoseconds and 2.5 nanoseconds, respectively.

the spontaneous emission factor, and $g$ is the optical gain of the stimulated emission. The third terms of the righthand side of both equations represent the efficiency of stimulated emission. $\tau_{c}$ depends on the PIN structure of the diode. It is typically a few nanoseconds. $\tau_{p}$ is determined by the character of the cavity as:

$$
\tau_{p}=\frac{\eta}{c}\left\{\alpha+\frac{1}{L} \ln \frac{1}{R}\right\}^{-1}
$$

where $\eta$ is the refractive index of the active region, typically $\sim 3.5, \alpha$ is the photon decay coefficient, $L$ is the length of the cavity, and $R$ is the reflectivity of the mirror in the cavity. The second term in the parentheses corresponds to internal absorption of the cavity. A typical value of the expression in parentheses is about $40 \mathrm{~cm}^{-1}$. Therefore, the photon lifetime is a few picoseconds, namely shorter than the lifetime of carrier:

$$
\tau_{\mathrm{c}} \gg \tau_{\mathrm{p}} .
$$

This character is an essential feature of a laser diode, which enables the high-speed response of intensity modulation.

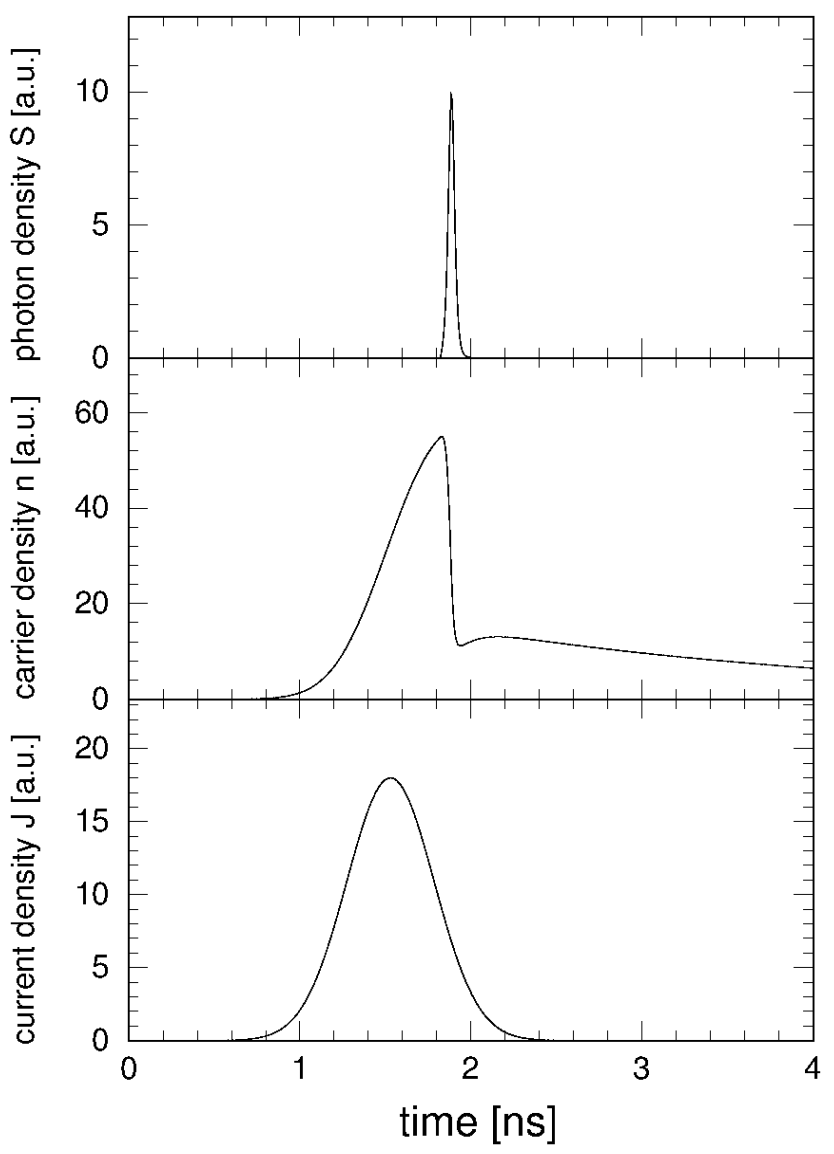

Fig. 2. Simulation of LD emission, similar to Figure 1. An electric pulse with 600 picoseconds in FWHM is applied. The pulse height is adjusted to induce the laser pulse after the peak of the electric pulse.

Time variations of $J, n$, and $S$ are calculated based on Equation (1) and (2) as shown in Figure 1. In this simulation, a step function is assumed for the electric current. After this is applied to the LD, the carrier density $n$ is increased continually, and photons are generated by spontaneous emission according to the first term of the right side of equation (2). Following this equation, $S$ is increased in proportion to $n$ by the spontaneous emission represented by the first term, reduced in proportion to $S$ by absorptions and scatterings in the cavity represented by the second term, and increased to the square of $S$ by the stimulated emission represented by the third term. At the beginning stage, the loses due to the second term are predominant. Eventually, the stimulated emission overwhelms these losses and photons fill the active region. At this stage, an avalanche phenomenon is induced, namely photons from a stimulated emission induce another stimulated emission. Due to this chain reaction, stimulated emission becomes dominant. Consequently, $S$ is increased exponentially, and $n$ falls rapidly. Then $S$ is rapidly reduced with a decay time of 
$\tau_{p}$ due to the deficit of $n$ according to the second term. As a result, a sharp laser pulse is emitted.

If the current continues to be applied after $S$ decreases, $n$ increases again, and the same process is iterated. This iteration is called "relaxation oscillation." After this oscillation, the photon density $S$ converges to a certain value.

Following the general feature of LD emission as explained above, we discuss a technique to extract the first laser pulse from the relaxation oscillation. If $J$ continues to be applied after the stimulated emission is started, $n$ continues to increase, and then the intensity of photon emission increases. As a result, the width of the first pulse of the oscillation becomes wider. If $J$ continues to be applied after the photon emission is decreased, $n$ is increased again as explained above.

Therefore, J should be stopped after the first laser pulse is emitted. To make the laser pulse shorter, $J$ should be reduced as soon as the stimulated emission is increased. Therefore, the pulse width of the applied current should be narrower than one nanosecond, and the integrated current density of the pulse should be slightly larger than $n_{t h} \times e$ to make the shortest pulse possible. The bias current, which is constantly applied to the LD, should be zero. A result of simulation with this configuration is shown in Figure 2. A 600 picoseconds electric pulse is applied in this simulation. The pulse height is adjusted to ensure that the first laser pulse is emitted after the peak of the electric pulse. As a result, the photon density $S$ forms into a sharp pulse with 60 picoseconds in FWHM.

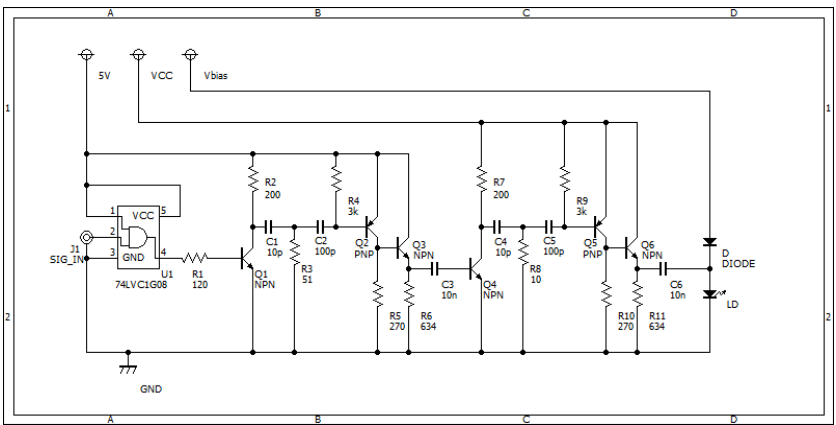

Fig. 3. A schematic diagram of the waveform shaping circuit in the pulsed laser. An external trigger signal is received by the connector indicated as "SIG_IN". The trigger signal is to be reshaped to a short pulse, which is applied to a LD. The height of the short electric pulse is variable by adjusting the input voltage indicated as Vcc.

\section{Pulse Generation Circuit}

In this section we describe the design of the short-pulsed laser developed in this study. The basic idea of this design came from reference [2]. As explained in the previous section, the pulsed laser is composed of an electric pulse generator and a LD. The width of the electric pulse must be shorter than one nanosecond and its height must be adjustable.

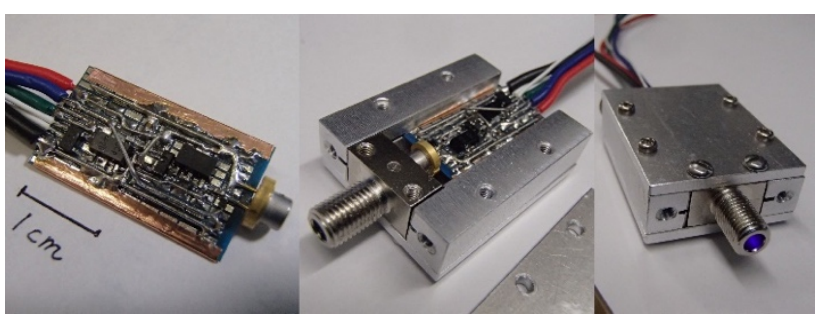

Fig. 4. Pictures of the pulsed laser. The pulse shaping circuit and the laser diode are contained in a small aluminum box, which also provides for thermal conduction. The aluminum box is designed to connect to an optical fiber.

In this pulsed laser, the electric pulse is generated by a simple shaping circuit. We adopted double differential circuits as shown in Figure 3 to generate an electric pulse with less than one nanosecond width. The first differential circuit is equipped with following three functions:

1. receive a trigger signal and convert it to a square wave,

2. transform the square wave to a short pulse, and

3. amplify the short pulse and send it to the following second differential circuits.

An external trigger pulse, $5 \mathrm{~V}$ TTL signal, is injected into a comparator and the comparator converts it to a square wave. The risetime of this comparator directly affects the width of the electric pulse generated. Therefore, a high-speed comparator should be adopted. Also, the pulse height of the square wave must be large enough to drive the following transistor. For these purposes we use a single 2-input AND gate comparator SN74VC1G08, of which the switching time is characteristically a few nanoseconds. The square wave goes through the collector of a high speed NPN transistor BFG591 denoted $\mathrm{Q}_{1}$ in Figure 3. This transistor amplifies and inverts the input square wave. Then the inverted signal is differentiated by an RC circuit which is composed of $R_{2}$ and $C_{1}$ in Figure 3 . The time constant of this differential circuit is $51 \Omega \times 10 \mathrm{pF}=510$ picoseconds, which is short enough compared with the risetime of the comparator. The direct-current component from transistor $\mathrm{Q}_{\text {in }}$ is removed by the capacitor $\mathrm{C}_{2}$ and the differentiated pulses go through the high speed PNP transistor BFT92 which is denoted $\mathrm{Q}_{2}$ in the figure. This transistor removes the positive pulse and inverts the negative pulse. Then the positive pulse, which has been inverted by the PNP transistor, is sent to the following NPN transistor, which is denoted $\mathrm{Q}_{3}$. This transistor, BFG591, amplifies the pulse with $15 \mathrm{~V}$ of collector-to-emitter voltage up to $2 \mathrm{~W}$.

The width of the electric pulse from the transistor $Q_{3}$ is about one nanosecond, which is limited by the response 
time of the comparator. This one nanosecond pulse is sent to the second differential circuit.

The second differential circuit is designed to make the electric pulse shorter. The sequence of this circuit is the same as the first one except for its decay constant, which is $10 \Omega \times 10 \mathrm{pF}=100$ picoseconds as shown in the figure. The FWHM of the reshaped electric pulse is 600 picoseconds. The amplitude of the electric pulse can be adjusted by changing Vcc indicated in Figure 3.

The electric pulse is sent to a LD. We adopted the Nichia NDV4212 for this light source, for which the wavelength is $405 \mathrm{~nm}$ and the intensity of emission is $120 \mathrm{~mW}$ at maximum. This LD can be replaced depending on the purpose of the pulsed laser.

The pulsed laser developed in this study is shown in Figure 4. The electric circuit is on a universal board of dimensions $20 \mathrm{~mm} \times 10 \mathrm{~mm}$. The LD is mounted on the board directly as well. This board is contained in an aluminum box, to which the LD is directly coupled for thermal conduction. This box is also designed to connect to an optical fiber.

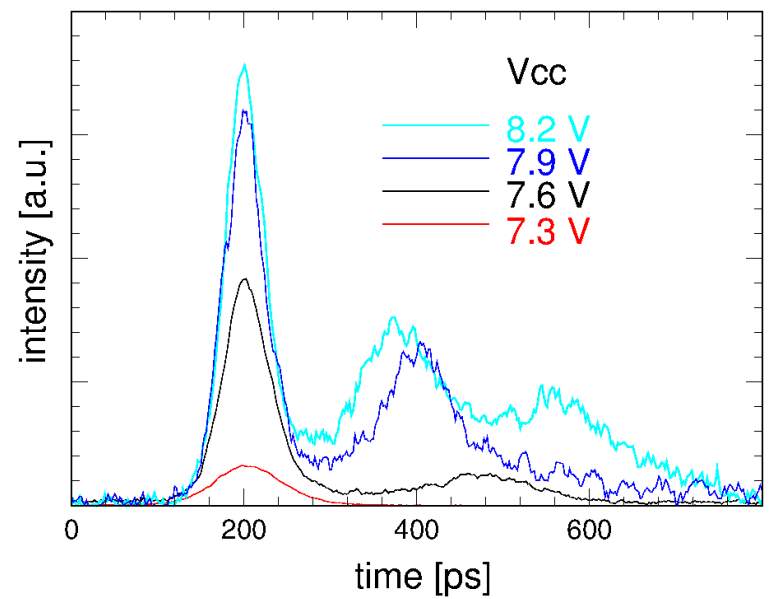

Fig. 5. Light curve of the laser pulses measured by a streak camera. The time resolution of the streak camera is two picoseconds. The amplitude of the applied electric pulses are adjusted by $\mathrm{Vcc}$ as indicated in the figure. Results from four Vcc values are shown. $7.3 \mathrm{~V}$ is the optimum voltage to extract the first pulse without relaxation oscillations. An 80picosecond pulse width in FWHM is achieved at this Vcc value. A higher intensity pulse is emitted with the relaxation oscillation when a higher Vcc is applied.

We measured the shape of the laser pulse from the LD using a streak camera (HAMAMATSU, C5680), of two picoseconds time resolution as shown in figure 5 . After adjustment of the amplitude of the electric pulse, a laser pulse with 80 picoseconds in FWHM was observed. With increasing electric pulse amplitude by changing $\mathrm{Vcc}$, the intensity of the laser pulse is increased with the relaxation oscillation as expected.

We also measured the stability of the intensity of the laser pulse. This pulse was transmitted through an optical fiber to a PMT. The pulse height of the signal from the PMT was monitored by an oscilloscope for 70 minutes starting immediately after the pulsed laser began emitting. The repetition rate of the laser pulse was one $\mathrm{MHz}$. As a result, the stability of the pulse intensity was $2.5 \%$ in one sigma deviation.

\section{Application to a PMT calibration}

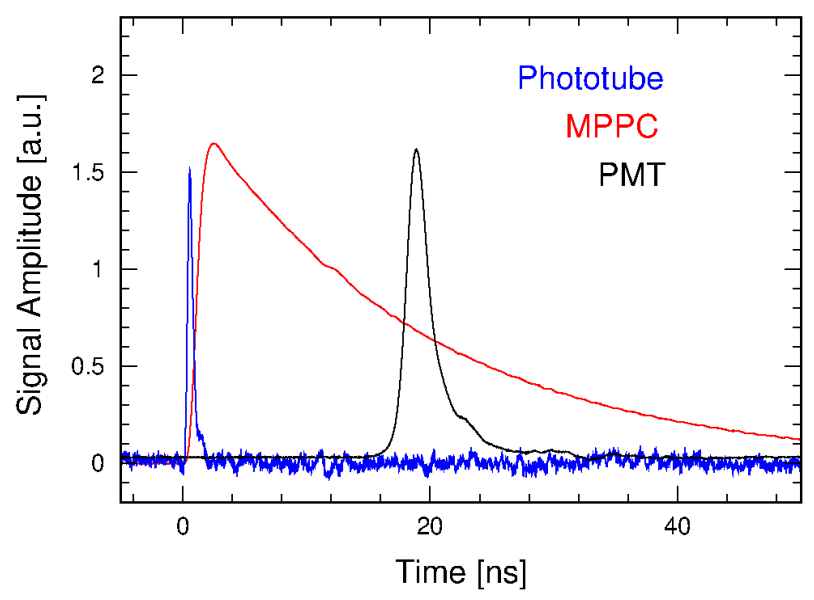

Fig. 6. Signal waveforms from photon detectors measured using the pulsed laser developed in this study. Average waveforms of 1000 events are shown. The vertical scales are adjusted to compare transit times of the detectors. The first peak is the signal from a phototube, the second one is from an MPPC, and the third one is a PMT.

Using the pulsed laser developed in this study, we have measured the transit time of photon-detectors. A PMT (HAMAMATSU R12992-100-20), an MPPC (HAMAMATSU S10362-33), and a biplanar phototube (HAMAMATSU R1328U) are used in this measurement. Transit time is defined as a time duration between the time of photons reaching the photo-cathode and the peak time of its signal pulse. In case of the PMT, the photoelectrons are multiplied in dynodes. Therefore, the transit time is much longer than its signal width. The width of the avalanche region of the MPPC is narrower than that of the PMT. Therefore, the transit time of the MPPC is much shorter. In the case of the phototube, photoelectrons are not multiplied at all, and the distance from photo-cathode and the anode is $2 \mathrm{~mm}$. Therefore, the transit time for the biplanar phototube is less than 100 pico-seconds which is equivalent to the pulse width of the laser. Figure 6 shows the pulse shape of these photon devices. The cathodes of three devices are located at the same distance from the laser. No preamplifier is used, and cable lengths are corrected. Averages over 1000 events are shown. The first peak in this figure is the signal from the phototube. Since the response time of this device is equivalent to the pulse width of the laser, the laser pulse cannot be considered as a delta function anymore. Also, this measurement is restricted by the bandwidth of the oscilloscope $(2 \mathrm{GHz})$. 
The start time, when the first photons arrived at the photo-cathode, is estimated from the transit time of this signal $\mathrm{TT}_{\mathrm{r}}$ which includes the transit time of the phototube, the risetime of the laser pulse and the bandwidth of the oscilloscope. We assume $\mathrm{TT}_{n}$ is $1 / 0.8$ times of 10 to $90 \%$ of rising time of the pulse [3]. In other words, the start time is estimated as the $\mathrm{TT}_{\mathrm{pt}}$ before the peak of the signal from the phototube. Based on this estimation, transit times of the MPPC and PMT are estimated as 2.5 nanoseconds and 18.9 nanoseconds, respectively with a systematic uncertainty of 100 picoseconds.

\section{Summary}

We have developed a short-pulsed laser which utilizes properties of the laser diode. The width of the laser pulse is 80 picoseconds, and its intensity is adjustable. This pulsed laser is composed of a simple and compact electrical circuit and a laser diode. The laser diode can be replaced depending on pulses desired. Examples of measurements of transit times from different photon detectors are shown. The pulse width of the laser beam is short enough to measure time properties of the photon detectors precisely.

\section{References}

1. Cherenkov Telescope Array Consortium: B. S. Acharya, I. Agudo, I. A. Samarai, R. Alfaro, J. Alfaro, C. Alispach, R. Alves Batista, J.-P. Amans, et al., Science with the Cherenkov Telescope Array, arXiv:1709.07997.

2. Wilfried Uhring, Chantal-Virginie Zinta \& Jeremy Bar-tringer: A low cost high repetition rate picosecondlaser diode pulse generator. Proc. SPIE 5452, Semi-conductor Lasers and Laser Dynamics, 1 September2004W.-K. Chen, Linear Networks and Systems (Book style). Belmont, CA: Wadsworth, 1993, pp. 123-135.M. Ben Rabha, M.F. Boujmil, M. Saadoun, B. Bessaïs, Eur. Phys. J. Appl. Phys. (to be published)

3. Hamamatsu Photonics K.K., "PHOTOMULTIPLIER TUBES, Basics and Applications", Hamamatsu Photonics K.K. 1994 